\title{
Diversity-Induced Resonance
}

\author{
Claudio J. Tessone, ${ }^{1,2}$ Claudio R. Mirasso, ${ }^{2}$ Raúl Toral,,${ }^{1,2}$ and James D. Gunton ${ }^{3}$ \\ ${ }^{1}$ Instituto Mediterráneo de Estudios Avanzados (IMEDEA), CSIC-UIB, Ed. Mateu Orfila, \\ Campus UIB, E-07122 Palma de Mallorca, Spain \\ ${ }^{2}$ Departament de Física, Universitat de les Illes Balears, E-07122 Palma de Mallorca, Spain \\ ${ }^{3}$ Department of Physics, Lehigh University, Bethlehem, Pennsylvania 18015, USA
}

(Received 3 May 2006; published 6 November 2006)

\begin{abstract}
We present conclusive evidence showing that different sources of diversity, such as those represented by quenched disorder or noise, can induce a resonant collective behavior in an ensemble of coupled bistable or excitable systems. Our analytical and numerical results show that when such systems are subjected to an external subthreshold signal, their response is optimized for an intermediate value of the diversity. These findings show that intrinsic diversity might have a constructive role and suggest that natural systems might profit from their diversity in order to optimize the response to an external stimulus.
\end{abstract}

DOI: $10.1103 /$ PhysRevLett.97.194101

PACS numbers: 05.45.Xt, 02.50.- $-\mathrm{r}$

Noise-induced, or stochastic, resonance emerged in the early 1980 s as a proposal to explain the periodicity observed in the Earth ice ages [1,2]. The mechanism is such that an external forcing acting upon a nonlinear system can be conveniently amplified under the presence of the right amount of noise. This innovative proposal led many researchers to look for a similar constructive role of noise in physical, chemical, biological, and many other kinds of systems [3-6]. While initially the studies focused on simple, low-dimension, dynamical systems, more recent work $[7,8]$ has considered the constructive role of noise in extended systems composed of many coupled identical units. The assumption of identical units, while being mathematically convenient, is not very realistic for many of the applications, since it is clear that in some natural systems, especially in biology, the units composing the ensemble present a disparity in the values of some characteristic parameters. Among other consequences, this natural diversity makes each isolated system respond differently to an external forcing; it is an open question to investigate the effect that diversity has on the global response of the collective system.

This problem has received some recent attention. For instance, Hong [9] analyzes the locking behavior of an ensemble of coupled oscillators with different internal frequencies subject to a periodic external forcing. He finds that the quenched disorder helps a small fraction of the oscillators to lock to the external frequency. However, he does not observe a collective behavior in which the whole ensemble benefits from the diversity in the internal frequencies. In this Letter, we give evidence that the right amount of diversity, in the form of quenched noise, might help an extended system to respond globally in a more coherent way to an external stimulus.

As in the case of stochastic resonance, we believe that the results reported here are very general. For the sake of concreteness, however, we have considered two prototypical nonlinear systems: one bistable and another excitable.
In both cases, we show that there is a resonance effect in the global response as a function of the diversity.

We consider first an ensemble of $N$ globally coupled bistable systems, whose dynamics is given by

$$
\dot{x}_{i}=x_{i}-x_{i}^{3}+a_{i}+\frac{C}{N} \sum_{j=1}^{N}\left(x_{j}-x_{i}\right)+A \sin (\Omega t) .
$$

Here $x_{i}(t), i=1, \ldots, N$, is the position of the $i$ th unit at time $t$, and $C$ is the coupling strength. The location and relative stability of the fixed points of the dynamics of an isolated unit $i$ are modified by the parameter $a_{i}$. We assume the $a_{i}$ 's to take independent values distributed according to a probability distribution function $g(a)$ that satisfies $\langle a\rangle=$ $0,\left\langle a_{i} a_{j}\right\rangle=\delta_{i j} \sigma^{2} . \sigma$ will be referred to as the diversity. The system is also subjected to an external periodic forcing, of intensity $A$ and frequency $\Omega=2 \pi / T$. For simplicity, and to emphasize the role of the diversity, we neglect standard noise effects on those equations.

In order to quantify the response of the system to the external forcing, we introduce the average position of the units $X(t)=\frac{1}{N} \sum_{i=1}^{N} x_{i}(t)$. In the globally coupled case considered here, the coupling among units appears only through this macroscopic quantity:

$$
\dot{x}_{i}=C X+(1-C) x_{i}-x_{i}^{3}+a_{i}+A \sin (\Omega t) .
$$

This can be put in a potential form $\dot{x}_{i}=-\left(\partial V_{i} / \partial x_{i}\right)$ with a suitable time-dependent potential $V_{i}\left(x_{i} ; t, a_{i}, X\right)$ whose explicit expression can be easily obtained. By averaging Eq. (2) over the whole population, we obtain

$$
\dot{X}=X-\frac{1}{N} \sum_{i} x_{i}^{3}+A \sin (\Omega t) .
$$

Here and henceforth, averages with respect to the variables $a_{i}$ are replaced with averages with respect to the distribution $g(a)$. Following Ref. [10], let us introduce $\delta_{i}$, such that $x_{i}=X+\delta_{i}$. We additionally introduce $\frac{1}{N} \sum_{i} \delta_{i}^{2}=M$. 
Notice that $M(t)$ is a function of time as it depends on the distribution of $\delta_{i}(t)$; furthermore, $M \geq 0$. Under the assumption of $\delta_{i}$ being distributed according to an even distribution[11], we get, using Eq. (3),

$$
\dot{X}=X(1-3 M)-X^{3}+A \sin (\Omega t) .
$$

The unforced system is bistable with equilibrium points at $X_{ \pm}= \pm \sqrt{1-3 M}$. For $\sigma=0, M$ vanishes after an initial transient to wash out the effect of the possibly different initial conditions for the $x_{i}$ 's. A weak, subthreshold forcing (namely, $A \lesssim 0.3$ for the range of frequencies used in this work) will not suffice to have the global variable $X$ jump from one stable point to the other as it will simply make small oscillations around one of the equilibrium points. As the diversity increases, $M$ increases with a twofold effect: first, the stable points approach each other, and, second, the height of the barrier separating them decreases. It might be possible that the weak external forcing is now able to overcome the reduced barrier and the global variable $X$ exhibits wide oscillations between the two fixed points following the external forcing. If the diversity increases even further, leading to $M>1 / 3$, the barrier disappears, the two fixed points merge at $X_{0}=0$, and the global variable makes small oscillations around this new fixed point. We then predict a resonance effect for intermediate values of the diversity for which the amplitude of the oscillations of $X$ will be maximum.

It should be clear now what the mechanism is leading to the resonance. In the homogeneous case, when all systems have $a_{i}=0$, the subthreshold forcing cannot overcome the potential barrier for any of them. As the diversity increases, there will be a number of units for which the value of $a_{i}$ is such that the forcing is now suprathreshold for them and the barrier can be overcome in one direction. These units are able, through the coupling term, to pull the other units and, hence, produce a collective, macroscopic movement following the variation of the external forcing. For too large diversity, however, some of the units to be pulled offer too much resistance to follow the external force, and this effect cannot be overcome by the favorable units.

Before we present the numerical results sustaining this diversity-induced resonance, let us present a simplified treatment that allows us to reproduce the aforementioned effect. The main problem to solve numerically Eq. (4), to determine the time evolution of the global variable $X(t)$, is to find the variation in time of the second moment $M(t)$. The classical treatment of Ref. [10] consists in writing down a hierarchy of equations which is truncated under some Gaussian approximations for the moments. We follow here an alternative approach. Using an ensemble average, we can write $M(t)=\int \operatorname{dag}(a)[x(t ; a)-X(t)]^{2}$, where $a$ is distributed according to the distribution $g(a)$ and $x(t, a)$ is the position at time $t$ of a particle whose diversity parameter takes the value $a$. This integral is performed numerically using a Gaussian quadrature scheme, for which we need to compute the necessary values of the function $x(t, a)$. This calculation is done in a regime of "slow forcing," where the period of the forcing signal is large enough such that, given a value of $X(t)$, $x(t, a)$ can be considered as the rest point given by the minimum of the local potential $V(x ; t, a, X)$ or the root of $d V / d x=0$. This cubic equation can have either one or three real roots: in the latter case, we selected the root with the lowest potential value [12]. Once $M(t)$ has been computed in this way, the right-hand side of Eq. (4) is fully determined, and we can proceed with its numerical integration to find the time evolution of $X(t)$. We quantify the resonance effect by the spectral amplification factor [13] $\eta=4 A^{-2}\left|\left\langle e^{i \Omega t} X(t)\right\rangle\right|^{2}$, where $\langle\ldots\rangle$ denotes a time average.

In Fig. 1, we plot the amplification factor $\eta$ versus the diversity $\sigma$, for different values of the period $T$ of the external forcing for an amplitude $A$ below the threshold value. As predicted, there is an optimum value of the diversity for maximum amplification, the main result of this Letter. Notice that our approximate treatment agrees rather well with the results coming from a direct numerical integration of the original set of Eq. (1), when the signal is slow.

We now analyze how the system responds to different modulation periods of the external forcing while the amplitude is kept fixed. In the inset in Fig. 1, we plot the amplification factor as a function of the period of the external forcing for fixed diversity. It can be seen that for large $T$ the amplification factor reaches a constant value, while $\eta$ vanishes for small $T$. Both regimes are well described by the theoretical approximation. For large $T$,

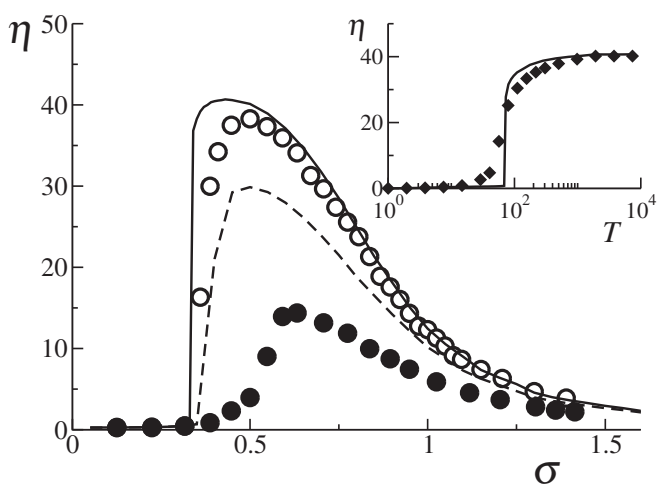

FIG. 1. Spectral amplification factor $\eta$ of the globally coupled bistable model, Eq. (1). The values of the $a_{i}$ 's are drawn from a Gaussian distribution of zero mean and variance $\sigma^{2}$. Some system parameters are $N=10^{3}, C=1, A=0.20$. In the main plot, we observe that the amplification factor exhibits a maximum as a function of the diversity in the case of both a period $T=50$ (black circles) and $T=10^{3}$ (open circles) of the external forcing. The inset plots the spectral amplification factor as a function of the period of the forcing (the diversity is fixed at $\sigma=$ $0.55)$. In both plots, symbols correspond to numerical simulations, and the lines are the corresponding theoretical predictions of a simplified theory (see the text for details). 
the agreement is due to the validity of our approximate picture of the dynamics in that limit. For small $T$, the individual units are not able to follow the fast external forcing, and, consequently, $\dot{x}_{i} \approx 0$, which leads to the same condition to determine $x_{i}$ as a function of $a_{i}$ and $X$ as in the large $T$ limit. It is worth mentioning that the shape of the curve in the inset in Fig. 1 differs from what appears in stochastic resonance where a maximum at intermediate values of $T$ is observed [4]. This difference is due to the absence in the diversity-induced resonance case of a matching between two time scales which in stochastic resonance are the Kramer's time and the forcing period.

In Fig. 2(a), we study the effect of the amplitude of the forcing on the system response. As in stochastic resonance [4], a maximum in the response appears only for subthreshold forcing, and the height of this maximum increases with decreasing amplitude. However, for suprathreshold forcing (the case $A=0.7$ ), the linear regime is recovered, and the amplification factor steadily decreases with increasing diversity. Figure 2(b) shows that the spectral amplification factor has a maximum for a well-defined value of the amplitude of the external forcing.

We now turn our attention to excitable systems. As a paradigmatic model of interest in many biological applications, we consider a globally coupled ensemble of excit-

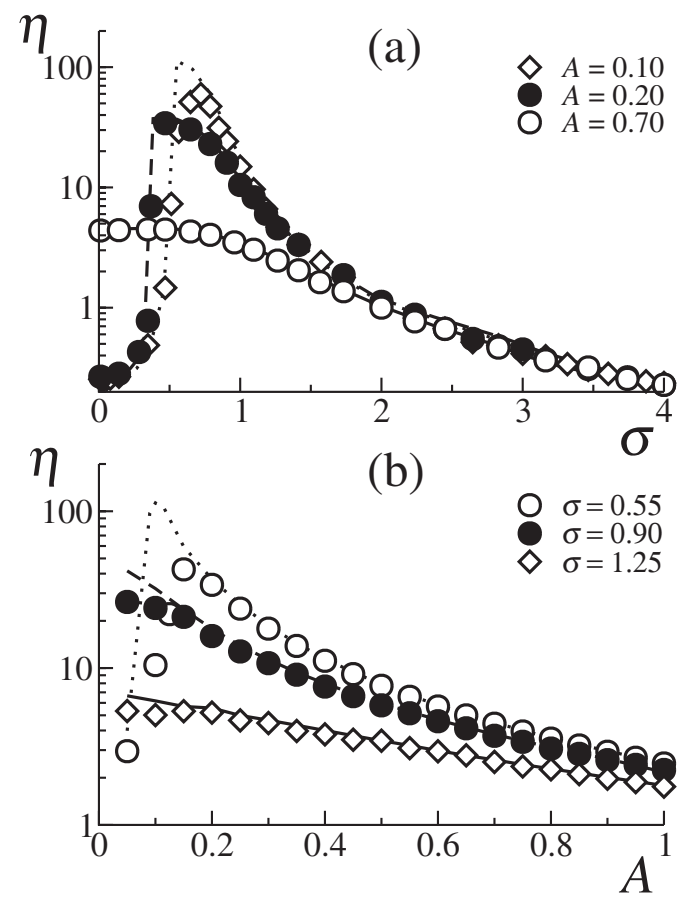

FIG. 2. (a) Spectral amplification factor $\eta$ of the globally coupled bistable model, Eq. (1) as a function of diversity for different values of the amplitude $A$ of the forcing. (b) Dependence of $\eta$ on the forcing amplitude for fixed values of diversity. Both figures use a period $T=200$ and other parameters as in Fig. 1. Symbols represent the results coming from a numerical simulation of the system's equations, and lines correspond to the analytical approximation (see text for details). able units described by the FitzHugh-Nagumo equations:

$$
\begin{aligned}
\epsilon \dot{x}_{i} & =x_{i}-\frac{1}{3} x_{i}^{3}-y_{i}+\frac{C}{N} \sum_{j=1}^{N}\left(x_{j}-x_{i}\right), \\
\dot{y}_{i} & =x_{i}+a_{i}+A \sin (\Omega t) .
\end{aligned}
$$

The coupling between units is taken into account through the activator variable $x$ with a coupling strength $C$. Each unit has a parameter $a_{i}$, representing the diversity, drawn from a probability distribution $g(a)$ of mean $\left\langle a_{i}\right\rangle=a$ and correlations $\left\langle\left(a_{i}-a\right)\left(a_{j}-a\right)\right\rangle=\delta_{i j} \sigma^{2}$. When $\left|a_{i}\right|<1$, system $i$ is in the oscillatory regime, while for $\left|a_{i}\right| \geq 1$ it is in the excitable one. As in the double-well case, the system is subjected to a periodic forcing of intensity $A$ and frequency $\Omega$, and we do not consider explicit noise terms. The combined effect of diversity and noise was considered in Ref. [14] in the context of coherence resonance. Specifically, the authors of this reference found that, in the unforced case $A=0$ and in the presence of noise, there was a systematic increase of the coherence factor for increasing inhomogeneity. We focus in this Letter on the forced case $A \neq 0$, where we will show a resonance effect with respect to the diversity.
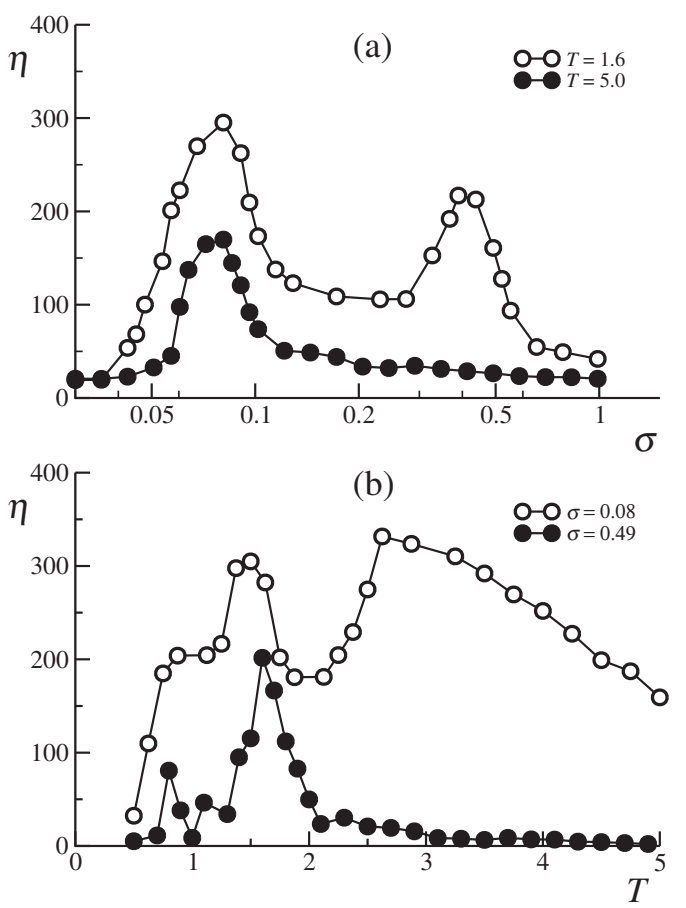

FIG. 3. Spectral amplification factor $\eta$ of the globally coupled FitzHugh-Nagumo model, Eqs. (5), where the $a_{i}$ 's have been drawn from a Gaussian distribution of mean $a$ and variance $\sigma^{2}$. Some system parameters are $N=10^{3}, \epsilon=10^{-2}, a=1.12, C=$ $1, A=0.05$. (a) Plot of $\eta$ as a function of the diversity $\sigma$ for different periods of the external forcing. (b) Plot of $\eta$ as a function of the period $T$ for different values of the diversity $\sigma$. In both cases, the symbols represent the results coming from a numerical simulation of the system's equations (the solid line is a guide to the eye). 
The theoretical analysis follows the lines of the doublewell system. With the definitions $X=\frac{1}{N} \sum_{i} x_{i}, Y=\frac{1}{N} \sum_{i} y_{i}$, and $M=\frac{1}{N} \sum_{i}\left(x_{i}-X\right)^{2}$, we arrive at

$$
\begin{aligned}
\epsilon \dot{X} & =X(1-M)-\frac{X^{3}}{3}-Y, \\
\dot{Y} & =X+a+A \sin (\Omega t) .
\end{aligned}
$$

We conclude that in this model an increase in the diversity, hence an increase in $M$, induces a change in the shape of the nullclines of the dynamics of the global variables. As in the double-well system, in the homogeneous case $a_{i}=a$ and $|a|>1$, all units are in the excitable regime, and we consider the case where the weak external forcing is not enough to overcome their excitability threshold. As the diversity increases, some units will have their excitability threshold lowered (they could even become oscillatory), and the forcing is now suprathreshold for them. Those units pull the others, so producing the observed collective behavior. The actual description of the collective behavior is somewhat more involved, since $M$ exhibits a periodic variation with time, and it has a maximum value when the collective variables $X$ and $Y$ are near the fixed point. In the limit of large $M$, the nullclines are modified such that the limit cycle disappears altogether.

In Fig. 3(a), we plot the amplification factor $\eta$ of the global $X$ variable as a function of the diversity $\sigma$ for different values of the external time period $T$ and a fixed value of the amplitude $A$ close to threshold, where we can observe the resonance effect. This plot shows some differences with the double-well system studied before, namely, the presence of several resonances at different values of the diversity. We speculate that this behavior has its origin in the existence of a well-defined refractory time in the dynamics of an isolated unit. Several resonance maxima can also be observed when plotting the amplification factor as a function of the period of the forcing for fixed diversity; see Fig. 3(b). A similar effect has been also reported for a single FitzHugh-Nagumo system in the presence of noise, and it is known as frequency-dependent stochastic resonance [15].

In conclusion, we have given evidence that diversity, in the form of quenched noise, can enhance and lead to a resonant effect for the response of an extended system to an external periodic forcing. The evidence has been given for two prototype systems, paradigmatic of bistable and excitable behavior, and, hence, we believe that the same resonance will appear in other more complicated systems. The mechanism of the phenomenon is particularly simple: At a given time, a fraction of the units are able to respond to the external forcing; those units, through the coupling terms, are able to pull the others into the direction of the force. For too large diversity, the favorable units cannot overcome the effect of the adverse ones. This resonance mechanism is very general, and it could appear in many fields.
A final remark is relevant here. Note that in Eq. (4) for the global variable $X$ the effect of the diversity appears only through the variable $M$ measuring the dispersion in the behavior of the dynamics of the individual units $x_{i}$. Therefore, the existence of a resonance effect for the optimal amplification of weak signals does not depend on the source of the disorder. The same effect could also be obtained in the presence of disorder induced by noise (stochastic resonance), by a nonregular network of connectivities, inhibitory couplings, etc.

The idea that different sources of diversity can produce a resonant effect leads us to speculate that the amount of diversity present in some biological systems has an important function. Diversity could have been evolutionarily tuned in order to enhance the detection of weak signals. Whether natural systems have taken advantage or not from this diversity-related effect is a question that, as in the particular case of stochastic resonance, has not yet a clear answer.

We acknowledge financial support by the Ministerio de Educación y Ciencia (Spain) and FEDER Projects No. FIS2004-5073 and No. FIS2004-953, the EU NoE BioSim No. LSHB-CT-2004-005137, NSF Grant No. DMR-0302598, and the G. Harold and Leila Y. Mathers Charitable Foundation. C.R.M. acknowledges the hospitality and support of Lehigh University.

[1] R. Benzi, A. Sutera, and A. Vulpiani, J. Phys. A 14, L453 (1981).

[2] C. Nicolis and G. Nicolis, Tellus 33, 225 (1981).

[3] Proceedings of the NATO Advanced Workshop on Stochastic Resonance in Physics and Biology, edited by F. Moss, A. Bulsara, and M. F. Shlesinger [J. Stat. Phys. 70, No. 1-2 (1993)].

[4] L. Gammaitoni, P. Hänggi, P. Jung, and F. Marchesoni, Rev. Mod. Phys. 70, 223 (1998).

[5] B. Lindner, J. García-Ojalvo, A. Neiman, and L. Schimansky-Geier, Phys. Rep. 392, 321 (2004).

[6] T. Wellens, V. Shatokhin, and A. Buchleitner, Rep. Prog. Phys. 67, 45 (2004).

[7] B. von Haeften, R. Deza, and H. Wio, Phys. Rev. Lett. 84, 404 (2000).

[8] C. van den Broeck, J. Parrondo, and R. Toral, Phys. Rev. Lett. 73, 3395 (1994).

[9] H. Hong, Phys. Rev. E 71, 021102 (2005).

[10] R. Desai and R. Zwanzig, J. Stat. Phys. 19, 1 (1978).

[11] Alternatively, one could simply neglect the third moment of the distribution of $\delta_{i}$, an assumption valid for small $\delta_{i}$.

[12] As a consistency check, we have verified that the ensemble average $\int \operatorname{dag}(a) x(t ; a)$ is equal to $X(t)$ with sufficient numerical precision.

[13] P. Jung and P. Hänggi, Europhys. Lett. 8, 505 (1989).

[14] C. Zhou, J. Kurths, and B. Hu, Phys. Rev. Lett. 87, 098101 (2001).

[15] E. I. Volkov, E. Ullner, A. A. Zaikin, and J. Kurths, Phys. Rev. E 68, 061112 (2003). 